\title{
Role of Advanced Electron Microscopy in Unraveling Complex Microstructure in Nanostructured Thermoelectric Materials
}

\author{
Songting Cai, Zhongzhen Luo, Mercouri Kanatzidis and Vinayak Dravid \\ Northwestern University, Evanston, Illinois, United States
}

Thermoelectric (TE) materials can enable direct conversion between thermal and electrical energies and can impact waster heat recovery [1]. The conversion efficiency is determined by dimensionless figure of merit, $Z T=S^{2} \sigma T / \kappa$, where $S$ is the Seebeck coefficient, $\sigma$ is the electrical conductivity, $\kappa$ is the total thermal conductivity, and $T$ is the absolute temperature. $\mathrm{PbTe}$, a simple cubic rock-salt structure, has been studied for decades due to their promising band structure and high thermoelectric performance. Moreover, due to the simple crystal structure, faceted nano-precipitates can be easily constructed via supersaturated doping/alloying. Such highly strained nanostructure can greatly enhance phonon scattering and suppress the lattice thermal conductivity [2]. Here we have systematically characterized nanostructures in PbTe based materials, which lead to significantly enhanced thermoelectric properties [3].

Figure 1(a) shows a typical annular bright field (ABF) STEM image of Pb0.98Ga0.02Te-5\%GeTe, where a significant amount of faceted second phase ranging from 20 to $150 \mathrm{~nm}$ with brighter contrast is seen inside of the grain of the PbTe matrix. Moreover, numerous dislocations are located around the precipitates. From the energy dispersive spectroscopy (EDS) mapping (Figure 1(b)) of a selected area in (a), the precipitates are rich in $\mathrm{Ga}$ and deficient in $\mathrm{Pb}$.

To reveal more crystallographic information and orientation relationship between the precipitates and matrix, conventional and high-resolution TEM analyses were also applied. Figure 1(c) shows a middlemagnification TEM image of one such precipitate embedded in the matrix. Translational Moiré fringes are present due to lattice misfit between the two phases. According to the zoomed-in view of a part of the interface in Figure 1(d), the lattice fringes between the two phases are coherent (endotaxy). The corresponding selected area electron diffraction (SAED) is shown in Figure 1(e), where the bright diffraction spots belong to the rock-salt PbTe phase along the [111] direction. 6 satellite spots are observed around every bright spot, with the brightest ones originating from $\mathrm{Ga}_{2} \mathrm{Te}_{3}$ along [111] zone axis. The presence of all other weaker satellite spots as well as the translational Morié fringes come from double diffraction.

As a result, the nanoscale $\mathrm{Ga}_{2} \mathrm{Te}_{3}$ precipitates, dislocations and point defects leads to intensified phonon scattering and significant suppressed lattice thermal conductivity of $1.13 \mathrm{Wm}^{-1} \mathrm{~K}^{-1}$, nearly $34 \%$ reduction compared to that of $\mathrm{Pb}_{0.98 \mathrm{Ga}} \mathrm{Ga}_{02} \mathrm{Te}\left(1.72 \mathrm{Wm}^{-1} \mathrm{~K}^{-1}\right)$ at $300 \mathrm{~K}[3,4]$. 


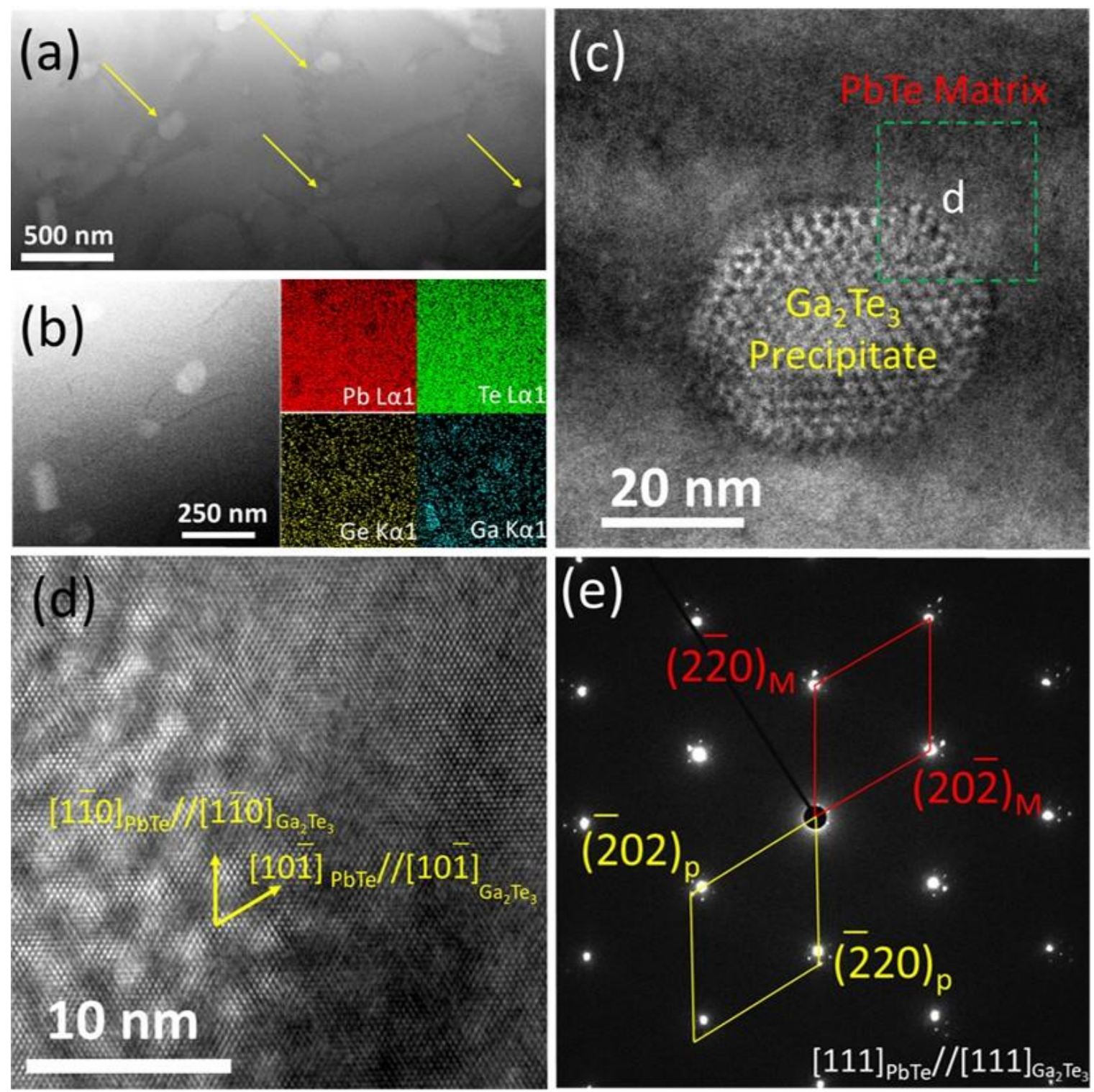

Figure 1. S/TEM analyses of Pb0.98Ga0.02Te-5\%GeTe. (a) Annular bright field STEM image. Decent amount of precipitates (indicated by yellow arrows) with brighter contrast are embedded in the PbTe matrix. The size of the precipitates is $\sim 20-60 \mathrm{~nm}$. (b) Zoom-in view of (a) along with EDS mapping. The precipitates are Ga-rich and Pb-deficient. (c) Typical TEM image of one precipitate embedded in the matrix. (d) Zoomed-in high-resolution TEM image of boxed area in (c), revealing a coherent phase boundary. (e) Corresponding selected area electron diffraction pattern (SAED) of (c) along the [111] direction of the PbTe matrix. The bright spots (labeled in red) belong to the PbTe phase, while the brightest satellite spots (labeled in yellow) were indexed to the Ga2Te3 phase (space group: F 43m) along the [111] direction. Other satellite spots are generated due to double diffraction. [3]

\section{References}

[1] Zeier, W. G. et al. Thinking Like a Chemist: Intuition in Thermoelectric Materials. Angewandte Chemie International Edition 55, 6826-6841 (2016).

[2] Cai, S. et al. Discordant nature of Cd in PbSe: off-centering and core-shell nanoscale CdSe precipitates lead to high thermoelectric performance. Energy \& Environmental Science 13, 200-211 (2020). 
[3] Luo, Z.-Z. et al. High Figure of Merit in Gallium-Doped Nanostructured n-Type PbTe- $x$ GeTe with Midgap States. J. Am. Chem. Soc. 141, 16169-16177 (2019).

[4] This work was supported by the Department of Energy, Office of Science Basic Energy Sciences under grant DE-SC0014520, DOE Office of Science. This work made use of the EPIC facility of Northwestern University's NUANCE Center, which has received support from the Soft and Hybrid Nanotechnology Experimental (SHyNE) Resource (NSF ECCS-1542205); the MRSEC program (NSF DMR-1720139) at the Materials Research Center; the International Institute for Nanotechnology (IIN); the Keck Foundation; and the State of Illinois, through the IIN. User Facilities are supported by the Office of Science of the U.S. Department of Energy under Contract No. DE-AC02-06CH11357 and DE-AC02-05CH11231. 